\title{
La holografía como recurso didáctico para la enseñanza de contenidos de geometría en primaria
}

\author{
Holography as an educational resource for the teaching \\ geometry content in primary school
}

Lorena Beteta-Serrano

Departament de Didàctica i Organització Escolar, Universitat de València, España lobese@alumni.uv.es

(D) José Eliseo Valle Aparicio

Departament de Didàctica i Organització Escolar, Universitat de València, España jose.e.valle@uv.es

\section{Ángel San Martín Alonso}

Departament de Didàctica i Organització Escolar, Universitat de València, España angel.sanmartin@uv.es

\section{RESUMEN}

En el presente artículo se analizarán los usos y beneficios que reportaría trabajar con hologramas como material didáctico, como así demuestran los estudios de Ghuloum, Escrivà y Orcos. A través de estas propuestas didácticas, se refuerza la idea de que el uso de la técnica de representación en tres dimensiones puede beneficiar al alumnado en la adquisición de los contenidos geométricos presentes en el currículum oficial. El ámbito de la geometría ofrece una amplia gama de contenidos que posibilitan trabajar mediante técnicas de representación gráfica. Cabe destacar, por otra parte, que ahora los estudiantes de educación primaria están socializados en el acceso y uso de imágenes de todo tipo. Sin duda alguna, su pensamiento visual está más evolucionado que el de generaciones anteriores, como ponen de manifiesto autores como Brea, Steiner, Gruzinsky o Mitchell. Con el manejo, tanto por parte del profesorado como del alumnado, de programas de diseño 3D y la utilización de la técnica holográfica del Fantasma de Pepper, se consiguen imágenes realistas de figuras con volumen y que a los estudiantes de primaria les interesa de modo especial. A partir de estrategias de observación, se ha podido constatar con algunos estudiantes que, cuando trabajan con estos recursos, mejoran considerablemente su dominio de las formas geométricas y los conceptos que las acompañan.

PALABRAS CLAVE enseñanza primaria, geometría, fotografía, proyecto de estudiantes, educación STEM.

\section{ABSTRACT}

In this article we propose to analyse the uses and benefits of working with holograms as educational resource, as evidenced by the studies of Ghuloum, Escriva, and Orcos. These didactic proposals reinforce the idea that the use of the three-dimensional rendering technique can benefit students in the acquisition of the geometric content present in the official curricu- 
lum. The scope of the geometry offers a wide range of contents that make it possible to work using graphical rendering techniques. It should be noted, on the other hand, that now elementary school students are socialized in the access and use of images of all kinds, without a doubt their visual thinking is more evolved than that of previous generations, as shown by authors such as Brea, Steiner, Gruzinsky or Mitchell. With the management of both teachers and students, 3D design programs and the use of Pepper's Ghost holographic technique, realistic images of volume figures are obtained and that elementary school students are particularly interested in it. Based on an observational methodology, we have been able to compare with some students who, when working with these resources, greatly improve their mastery of geometric shapes and the concepts that accompany them.

KEYWORDS primary education, geometry, photography, student projects, STEM education.

\section{INTRODUCCIÓN}

El uso de las tecnologías en las aulas es un hecho bastante extendido, ya que los centros docentes están dotados de recursos tales como Pizarras Digitales Interactivas (PDI), ordenadores, tabletas y/o ordenadores personales. La multitud de formatos y su adaptabilidad ofrecen una gran variedad de recursos llamativos que las convierten en herramientas singulares. Además, las nuevas generaciones, aquellas que han crecido en contacto con las tecnologías y a las que Prensky denominó como “nativos digitales” (Prensky, \& González, 2018), parecen sentirse más predispuestas a trabajar contenidos mediante el uso de estas herramientas.

Tanto el interés como la motivación del alumnado ante el estudio de contenidos es fundamental para "provocar aprendizajes significativos en los estudiantes, de manera interdisciplinar y holística” (Sánchez et al., 2020, p. 142). Así pues, el uso guiado de este tipo de metodologías no solo ayudaría a desarrollar su competencia tecnológica, sino que fomentaría la adquisición de conceptos trabajados de manera significativa y globalizada. Del mismo modo, y tomando como referencia las teorías constructivistas, estas apuntan que el alumnado ha de tener un papel protagonista en el proceso de enseñanza ya que solo así alcanzará un aprendizaje significativo.

Así pues, siguiendo la teoría constructivista de Piaget y el aprendizaje significativo de Ausubel, se pretende recoger sus principios para aplicarlos de manera oportuna al desarrollo del proyecto. Ahora bien, ¿cuáles son esos principios? Entre ellos, se destacan el aprendizaje significativo, la motivación, el principio de gradualidad, la experimentación y la actividad. Siguiendo a Agra et al. (2019), se entiende el aprendizaje significativo como aquel que pretende relacionar conocimientos previos con los actuales, asegurando la construcción del conocimiento mediante una metodología activa, donde el niño es protagonista de su propio aprendizaje y no un receptor pasivo de conocimiento, como apuntaba Locke con su tabula rasa. Del mismo modo, durante el proceso de enseñanza-aprendizaje es necesario que el niño esté motivado y tenga interés en aprender, ya que de este modo estará más predispuesto a trabajar y alcanzar los aprendizajes y conocimientos propuestos. Resaltar que estos siempre seguirán el principio de gradualidad inherente a la metodología constructivista, es decir, se presentarán estos de menor a mayor complejidad.

A través de las diferentes propuestas, los aprendizajes se irán construyendo como resultado de la actividad y la experimentación, siempre y cuando se desarrollen en un clima motivador. En este sentido el ámbito STEM (proviene de las siglas en inglés “Science, Technology, Engineering and Mathematics”, refiriéndose 
en español a "Ciencia, Tecnología, Ingeniería y Matemáticas") ofrece una gran variedad de contenidos y muchos de ellos podrían ser abordados mediante el uso de las tecnologías de la información y la comunicación en la enseñanza. Una de esas tecnologías disponibles son los hologramas que se pueden realizar mediante programas de diseño 3D. El manejo de este recurso didáctico contribuirá indudablemente a mejorar la adquisición de los contenidos de geometría, así como a potenciar las habilidades relacionadas con la representación visual y artística, además de fomentar la competencia matemática y la digital. A partir de este supuesto, el problema de investigación que se aborda en este trabajo es el diseño y evaluación de un holograma creado para la enseñanza de algunas figuras geométricas en sexto de Educación Primaria.

\subsection{Las tecnologías como agentes motivadores del aprendizaje en el aula}

Las niñas y niños tienen hoy acceso a los dispositivos tecnológicos en su entorno más próximo y, como apuntan Jiménez y Diez-Martínez (2018), aunque la mayoría de estos no se hayan diseñado con un fin didáctico, se pueden utilizar con este propósito. De ahí que no deban descartarse los recursos digitales antes de ser evaluados, sobre todo si se hace con el argumento de no tener una finalidad didáctica como objetivo principal. Es evidente que la gran diversidad de formatos y plataformas disponibles facilitan su adaptación a las características del alumnado y del proyecto que se pretenda desarrollar. Es tarea del personal docente hacer una selección de las diferentes herramientas y proporcionar al alumnado aquellas que encajen más con el objetivo a conseguir (González, \& Cerezo, 2020).

Ahora bien, ante la incorporación de estos recursos al aula, el alumnado ha de sentirse motivado hacia ellos, por lo que es conveniente destacar que los alumnos han de conocer por parte del personal docente no solo el qué, sino cómo, por qué y para qué (Sancho, 2017). En definitiva, darle sentido al proceso de aprendizaje para que el alumnado comprenda este y se involucre de manera más activa en él. Hemos de asumir que los niños y las niñas son nativos digitales, como mantienen San Martín y Peirats (2018), "el alumnado lleva a las aulas sus habilidades y aprendizajes adquiridos a través del manejo temprano de la tecnología digital" (p. 7). Experiencia que se deberá tener en cuenta a la hora de planificar las sesiones para partir desde el nivel de conocimiento que el alumnado posea de las herramientas y los contenidos, con la finalidad de mantener su interés ante los conceptos propuestos.

\subsection{Interrogantes en torno al uso de hologramas en la enseñanza de contenidos matemáticos}

Una de las primeras cuestiones a plantearse tiene que ver con las capacidades que se fomentan mediante la implementación del proyecto de holografía, ya que se van a trabajar contenidos matemáticos y de geometría. El ser humano siempre ha pretendido hacer una representación lo más fiel posible de la realidad en la que se encuentra, ya fuera a través de la palabra escrita o bien mediante imágenes. En relación con esta última, la pintura no llegó a hacer uso de más de una dimensión hasta el siglo XV, momento en el que se introdujo el concepto de perspectiva y con ella la profundidad de las obras (Kempt, 2000).

Pero ¿por qué hablar de perspectiva y dimensiones? Fundamentalmente porque aquí se maneja la holografía como estrategia metodológica para adquirir conceptos de geometría en la etapa de Educación Primaria. Por este motivo se menciona la perspectiva, ya que la holografía sería la evolución de esta al incorporar en la presentación de la realidad la tercera dimensión. Etimológicamente la palabra "holograma" 
está compuesta por dos términos griegos: holos, cuyo significado vendría a ser todo y grama que significa escritura (Ghuloum, 2010). Para Orcos (2017), el holograma se describiría como la reproducción "en tres dimensiones que proporcionan una sensación de realidad similar a la que percibimos con nuestros ojos cuando miramos a la realidad" (p.4).

El holograma puede presentarse como una técnica fotográfica basada en la refracción de imágenes que crean en conjunto una imagen tridimensional. Ahora bien, la técnica que se pretende desarrollar en estas páginas es conocida por el nombre del Fantasma de Pepper. Esta técnica tiene su origen en eventos recreativos, y consiste en reflejar una imagen a través del uso de luces en un vidrio, de modo que la imagen se visualiza en dicho cristal de forma poco sólida, con un aspecto fantasmal, de ahí el nombre de dicha técnica. Para aplicar esta técnica utilizando dispositivos digitales, es necesario que estos dispongan de pantallas que reflejen la imagen en tres dimensiones. En este sentido los prismas, para Orcos (2017) "se basan también en la misma técnica del Fantasma de Pepper, con la diferencia de que solo se coloca una superficie reflectora encima de un monitor o pantalla de manera que la imagen se ve en la superficie” (p. 5).

Dado que las tabletas u ordenadores pueden operar con diferentes programas de software libre, se pueden utilizar estas para realizar representaciones holográficas digitales. Y esta accesibilidad facilita el que sean los mismos alumnos y alumnas quienes elaboren sus propias figuras geométricas $y$, en consecuencia, los hologramas que de ellas se deriven.

De manera que una respuesta a por qué recurrir a la holografía como recurso didáctico es, sin duda, porque representar los contenidos geométricos mediante un holograma proporciona una imagen volumétrica que a los estudiantes les resulta muy atractiva y motivadora por su alto poder de ilustración y de interactividad, según constatan Ribeiro et al. (2018). Por su parte, Orcos et al. (2018) "cuando se usan diagramas o representaciones visuales se observa una mejora en el proceso de aprendizaje, ya que estos hacen que los alumnos presten atención a las relaciones que hay entre los distintos elementos" (p.93). Del mismo modo, en Escrivà et al. (2018) apuntan la necesidad de realizar representaciones visuales en la enseñanza de las matemáticas en la etapa de Educación Primaria.

Para Orcos et al. (2018), el holograma es una herramienta motivadora por sí misma, ya que presenta la imagen de objetos reales en movimiento. Esto produce una predisposición por parte del alumnado hacia el aprendizaje, ya que siente que este está realmente presente y que puede trabajar con y en él, motivando al alumnado desde edades tempranas en conceptos relacionados con los contenidos STEM y especialmente con los más abstractos de geometría. Además, la holografía contribuirá a formar el "ojo geométrico" de los estudiantes, competencia decisiva para la comprensión de los conocimientos más avanzados que requieran la capacidad de visualizar espacios y formas (Katsioloudis, \& Jones, 2018), siendo esto fundamental para su predisposición hacia el estudio de materias relacionadas en etapas superiores.

En esta misma línea, se asume que el uso de las tecnologías, al igual que las plataformas como medio para la realización de la técnica holográfica, se adapta al ritmo de los alumnos y a sus características. Por un lado, porque tienen acceso a los programas desde cualquier dispositivo; y, por otro, este tipo de proyectos presentan la información de forma más visual e icónica, con lo que contribuyen a mejorar la adquisición de determinados conceptos que de otra forma podrían no ser asimilados, en particular cuando el alumnado presente alguna dificultad en la comprensión de conceptos.

En cuanto al porqué se utiliza la técnica holográfica, se ha de señalar que al ser creadas las imágenes 3D por los discentes, hace obligatorio que el alumnado aplique lo aprendido, ya que ha de tener interiorizadas las figuras, así como los ejes y giros que produce el holograma. 
Respecto a la pregunta referida al para qué usar la holografía en el trabajo sobre los conceptos matemáticos, destacar que se ha de fomentar esa predisposición al estudio de contenidos abstractos en edades tempranas con el fin de favorecer su estudio en etapas posteriores. Así pues, el trabajo con hologramas podría ser una buena vía en este sentido. Gracias a su uso, los conceptos matemáticos podrían estar dotados de significado con el fin de que, en etapas posteriores, como se apunta en Torres (2020), el alumnado se sienta predispuesto a trabajar los contenidos de la materia.

A partir de los trabajos de Serra et al. (2009), entre otros, cabe resaltar que el holograma posee diversas funciones didácticas como son la cognoscitiva, la comunicativa, la informativa, la motivadora y la integradora, funciones todas ellas implicadas en el desarrollo integral del alumnado.

De este modo, la aplicación de la técnica holográfica del Fantasma de Pepper hace que los alumnos y las alumnas tengan que desenvolverse en diversas herramientas y plataformas digitales que, en principio, no fueron diseñadas con el fin de crear hologramas digitales y que, por tanto, podrán ser utilizadas con otros fines didácticos. Es decir, estas herramientas digitales serán útiles para el alumnado no solo durante el estudio de figuras geométricas, sino que también podrán hacer uso de ellas en otros ámbitos.

En definitiva, mediante el uso de la técnica holográfica el alumnado además de ser receptores, ellas y ellos serán quienes apliquen lo aprendido actuando propiamente como generadores de conocimientos.

\section{METODOLOGÍA}

El desarrollo metodológico adopta la estructura de un proyecto de experimentación centrado en un caso, ya que, mediante la observación de un alumno con dificultades de aprendizaje, se observó que este estaba más predispuesto a trabajar conceptos matemáticos si se hacían mediante las tecnologías. El estudio se basa en el trabajo con cuerpos geométricos mediante la holografía, respaldado en el uso de las TIC y la metodología basada en proyectos. Este enfoque de trabajo permite presentar al alumnado una visión más cercana al mundo que nos rodea, sin fragmentar el conocimiento en asignaturas como apunta Torres (2015) "un tipo de bloques de contenidos cuya significatividad y relevancia aparezcan ya a primera vista como motivadoras" (p. 100).

Más concretamente, se plantea el trabajo de conceptos geométricos, mediante el uso de una propuesta didáctica basada en unas determinadas tecnologías. Indudablemente, el desarrollo del proyecto, dado su planteamiento globalizador, implicará a otras materias como la informática, la educación plástica y visual o las ciencias naturales. Es necesario apuntar que la recientemente instaurada Ley Orgánica 3/2020, de 29 de diciembre, fija dentro de su artículo 19 los principios pedagógicos señalando que:

Sin perjuicio de su tratamiento específico en algunas de las áreas de la etapa, la comprensión lectora, la expresión oral y escrita, la comunicación audiovisual, la competencia digital, el fomento de la creatividad, del espíritu científico y del emprendimiento se trabajarán en todas las áreas (p. 122888).

Se ha optado por los conceptos geométricos debido a que no aparecen únicamente en el ámbito de las matemáticas, ya que están presentes en otras asignaturas como pueden ser la educación plástica y visual, y a la facilidad con la que el alumnado puede trabajar con estos conceptos mediante diversas técnicas. Además, los contenidos geométricos ayudan a comprender la realidad en la que vivimos. De hecho, gracias a 
la adquisición del concepto de volumen entendemos las dimensiones, su diferencia con las figuras planas, los poliedros, etc., en definitiva, todo lo que nos rodea en nuestro día a día.

Se debe señalar que el trabajo de este tipo de conceptos matemáticos se desarrollará a partir del segundo ciclo de la Educación Primaria, ya que es en este dónde se comienzan a trabajar los conceptos relacionados con los cuerpos geométricos. Dado que el proyecto se desarrolló en la Comunidad Valenciana, basamos los contenidos a trabajar en el Decreto 108/2014 de 4 de julio, los cuales presentan en el área de las matemáticas del siguiente modo:

TABLA 1. Contenidos del área de matemáticas y geometría en el Decreto 108/2014

\begin{tabular}{cl}
\hline CURSO & \multicolumn{1}{c}{ CONTENIDO } \\
\hline $3^{\circ}$ & $\begin{array}{l}\text { Elementos básicos: vértices, caras y aristas. } \\
\text { Vocabulario: prismas, pirámides, cilindro, cono } \\
\text { y esfera. }\end{array}$ \\
\hline $\mathbf{4}^{\circ}$ & $\begin{array}{l}\text { Elementos básicos: vértices, caras y aristas. } \\
\text { Prismas, pirámides, cilindro, cono y esfera. }\end{array}$ \\
\hline $5^{\circ}$ & $\begin{array}{l}\text { Cuerpos redondos: cono, cilindro y esfera. Prismas } \\
\text { y pirámides. Poliedros. } \\
\text { Elementos básicos: vértices, caras y aristas. } \\
\text { Desarrollo plano de prismas y pirámides. } \\
\text { Utilización del sistema de coordenadas cartesianas. }\end{array}$ \\
\hline $6^{\circ}$ & $\begin{array}{l}\text { Poliedros. } \\
\text { Elementos básicos: vértices, caras y aristas. } \\
\text { Poliedros regulares. Representación elemental } \\
\text { del espacio. Escalas. }\end{array}$ \\
\hline &
\end{tabular}

Se pretende dar aquí un enfoque alternativo al habitualmente mostrado por los libros de texto, donde se presenta la geometría mediante ilustraciones en dos dimensiones que no motivan al alumnado. Aunque normalmente se ha acompañado siempre al estudio geométrico con representaciones de figuras tridimensionales elaboradas por el alumnado con el fin de mostrar de manera visual y táctil los conceptos a trabajar, con esta propuesta se pretende trabajar estos conceptos haciendo uso de plataformas y herramientas digitales de cara a obtener representaciones tridimensionales.

\subsection{Objetivos}

El problema de estudio se define como diseñar y evaluar un proyecto de enseñanza de geometría mediante la aplicación de la holografía en sexto curso de Educación Primaria en un centro de la Comunidad Valencia.

La implementación se desarrolla orientada por los objetivos que se especifican a continuación.

TABLA 2. Definición de objetivos generales y específicos del proyecto

OBJETIVOS GENERALES OBJETIVOS ESPECÍFICOS

a) Revisar el estado de la cuestión

Investigar los referentes y conceptos del aprendizaje significativo.

Revisar los conceptos manejados en las publicaciones sobre aplicaciones anteriores de la holografía en la educación.

b) Diseñar el proyecto basado en la holografía Aplicar herramientas TIC de holografía en el desarrollo del proyecto.

Ofrecer estrategias a docentes y alumnado en el manejo de la holografía como recurso didáctico.

c) Evaluar la implementación del proyecto 
Así pues, entendemos la finalidad del proyecto como una alternativa de trabajo a la hora de abordar los conceptos geométricos. En concreto, se intenta, mediante la holografía, buscar una manera más llamativa y realista de presentar este tipo de contenidos, haciéndonos valer de las herramientas que proporcionan las Tecnologías de la Información y de la Comunicación (TIC).

\subsection{Participantes en el proyecto}

El diseño del proyecto de experimentación, su implementación y evaluación fueron realizadas por una profesora y un alumno de sexto curso de Educación Primaria de un centro público de una población próxima a la ciudad de Valencia. En todas las fases de trabajo la implicación y coordinación ente la docente y el estudiante fue total, participando tanto en las etapas de diseño como luego en la ejecución. De este modo el estudiante tuvo que realizar un holograma que sirvió para anotar las observaciones en el cuaderno de campo, tanto de sus dudas, como de sus iniciativas de ensayo/error.

\subsection{Fases en el desarrollo del proyecto}

1. Observación de un caso concreto: comenzando por la observación de un alumno con dificultades de aprendizaje en el área de las matemáticas, se llegó a la conclusión de que estaba más predispuesto a trabajar en esta asignatura si los contenidos se desarrollaban de manera lúdica y con recursos digitales.

2. Planteamiento de la hipótesis: se formuló la hipótesis de que las herramientas que ofrecen las TIC posibilitan la adquisición significativa de conocimiento.

3. Búsqueda de tecnología poco especializada: los investigadores comprobaron, mediante su uso, que existieran herramientas tecnológicas poco especializadas para que el alumnado pudiera trabajar con estas.

4. Concreción de los objetivos generales.

5. Revisión bibliográfica de trabajos anteriores sobre aprendizaje significativo y aplicaciones de la holografía en educación.

6. Concreción de los objetivos específicos del proyecto.

7. Descripción de las fases del proyecto: en concreto se establecieron un total de ocho fases para la realización total del proyecto, comenzando con la formación del profesorado, el proyecto holográfico en sí y terminando con la evaluación de dicho proyecto por parte de los agentes implicados en él.

8. Elaboración de materiales adaptados al nivel para el que se elaboró el proyecto, es decir, $6^{\circ}$ curso de Educación Primaria.

9. Prueba de los materiales: se realizó el proyecto holográfico con un alumno con dificultades de aprendizaje y se recogieron de datos adquiridos para este estudio mediante la observación directa y el registro por parte de los investigadores.

10. Ajuste de materiales: se reajustaron los materiales al no ser todas las plataformas elegidas aptas en todos los soportes informáticos. Por ello, nos decantamos por programas de elaboración en línea con acceso gratuito. 
11. Extraer conclusiones: una vez corregido el problema de las plataformas y ajustado las fases del proyecto, se evaluó el proceso del proyecto en sí mediante la observación y registro de las impresiones de los investigadores. Dichas impresiones quedan recogidas en el apartado de conclusiones de este artículo.

Dado que la aplicación del proyecto está destinada a llevarse a cabo en el ámbito de un centro escolar, la metodología a aplicar será descriptiva. De esta forma se pretendía registrar lo que ocurriría durante la aplicación del proyecto, basando los datos en un caso concreto y bajo unas características determinadas. Así pues, este proyecto puede tener diferentes resultados según el centro en el que se lleve a cabo y las características ambientales de este. Además, al darse en un medio no controlado por el investigador o investigadora los resultados esperados pueden variar de los propuestos en el inicio del proyecto.

\subsection{Recursos generados y utilizados durante el proyecto}

Como requisito para la puesta en marcha del proyecto fue necesario generar instrumentos de análisis y una serie de materiales didácticos entre los cuales se destacan:

1. Tutorial para docentes ${ }^{1}$ : vídeo explicativo del proceso completo de la formación del holograma. Utilizando como recursos ordenadores y tabletas, así como plataformas de acceso libre como SketchUp y Presentaciones de Google, se llevó a cabo un holograma de un cubo mediante la técnica del Fantasma de Pepper.

2. Plantilla para Presentaciones de Google: en dicha plantilla se establecía la imagen sobre la que tenían que incorporarse las figuras geométricas y en qué posición debían estar estas para que a la hora de reproducirla formara la figura en 3D.

3. Plantilla molde de acetato: molde para realizar el prisma que permite la visualización de la figura 3D.

4. Rúbrica de evaluación de contenidos curriculares: rúbrica de evaluación propuesta para el alumnado, dividida en las tres áreas trabajadas: matemáticas, ciencias naturales y educación plástica y visual; así como los contenidos generales de las tres materias.

5. Rúbrica de evaluación de plataformas digitales: para registrar los avances en las aplicaciones utilizadas, la asimilación de conceptos y el trabajo en equipo.

\section{RESULTADOS}

El proyecto se ha realizado, dadas las condiciones particulares del curso escolar, sin la posibilidad de acceder a ningún centro, con un único alumno de sexto curso, el cual no presentó ningún problema a la hora de realizar las fases de construcción del holograma. De hecho, el alumno, pese a presentar problemas en la compresión escrita, gracias a la realización del trabajo con hologramas fue constructor de su propio aprendizaje, corrigiendo los procesos cuando era necesario solventar algún pequeño fallo e incluso proponiendo nuevas técnicas holográficas. En concreto, propuso hacer una secuencia fotográfica con un elemento sólido sobre fondo negro y luego pasar estas fotos a vídeo para elaborar el holograma con movimiento. 
Además, el uso de programas no especializados permitió al alumno interactuar con ellos de forma fácil aplicando los tutoriales de cada plataforma. Durante el proceso de aplicación en relación a las plataformas solo se dio un problema, y es que uno de los programas propuesto al inicio no se podía trabajar con las tabletas digitales. De ahí que se decidiera cambiar las plataformas con las que trabajar haciendo que todas ellas fueran de carácter gratuito y en línea para que no existiera ningún inconveniente con el acceso. Como apuntan Moncada y Torres (2016), el aprendizaje se ha generado a partir del ensayo y del error.

Para la evaluación final se propusieron dos rúbricas de evaluación. Para Navarro Rodríguez et al. (2018) una rúbrica se consideraría "una guía de puntuación con criterios especificados que se utiliza para interpretar el trabajo del alumnado, cuya utilización facilita la corrección y objetividad en los aprendizajes que se pretenden alcanzar" (p. 84). Se seleccionaron, como plantea Cano (2015), las rúbricas en función de la sencillez de aplicación, además de que marcan las pautas a seguir durante el proyecto, pueden ser adaptadas y diseñadas de manera conjunta por profesorado y alumnado, así como que, al fijar los objetivos, se puede corregir el proyecto si este no se ajusta a las demandas.

Así pues, en referencia a la evaluación propuesta para el proyecto, cabe destacar que desde el primer momento se ha de saber qué conocimientos posee el alumno o alumna, con el fin de no repetir conceptos que ya ha asimilado y mantener su interés en la etapa inicial del proyecto. En este sentido, con la aplicación del proyecto, ya que estaba adaptado a las características y capacidades del alumno que lo desarrolló, este no perdió el interés durante la realización del mismo pues, como ya hemos comentado, el alumno propuso incluso un nuevo modelo de producción de hologramas. Así pues, gracias a estas aportaciones por parte del alumno y la evaluación final del proceso, se encontraron puntos de mejora para futuras aplicaciones del proyecto.

\section{DISCUSIÓN Y CONCLUSIONES}

La presencia de la holografía en las aulas hace de la implementación de este proyecto algo atractivo pedagógicamente y que, como hemos observado, se mantiene en muchos estudios académicos ya que proporciona ventajas en términos de aprendizaje en geometría y STEM. Apuntamos esto debido a que supone poner en el centro del proceso de enseñanza-aprendizaje no solo a las TIC, sino al alumnado como sujeto creador de su aprendizaje teniendo en cuenta sus características y convirtiendo así el proceso de enseñanza en individualizado.

Una de las enseñanzas extraídas de la implementación de este proyecto es que resulta fundamental que el personal docente seleccione las herramientas más útiles para las capacidades que se propone desarrollar en los niños y las niñas. Más útiles en el sentido de que sean las que mejor encajen con el objetivo propuesto y que sean capaces de adaptarse a las características individuales del alumnado. Por ello, es importante señalar que, como se ha demostrado durante este proyecto, se pueden utilizar programas y plataformas que no estuvieran destinadas o pensadas en sus inicios para fines educativos, pero que adaptándolos podemos encontrarles una utilidad didáctica.

No es necesario que los dispositivos tecnológicos y programas estén pensados desde la lógica de la didáctica para que puedan aplicarse con éxito en las aulas escolares, pero esto será así siempre y cuando el profesorado los adapte tanto a la intencionalidad educativa como a las características y necesidades de su alumno. Esta apertura de posibilidades respondería a la alternativa que propone Tonucci (2005) en relación con la for- 
mación fragmentada y especializada, señalando que "los frecuentes cambios en las tecnologías y de los procesos productivos, que requieren una formación más creativa, más abierta, más dúctil” (p. 47). En definitiva, recurrir a herramientas no especializadas, así como trabajar los contenidos mediante proyectos que engloben a diversas áreas presentando los contenidos como se dan en la realidad, es decir, de manera relacionada.

Siguiendo con el personal docente y en relación con esta propuesta, se debe hacer hincapié en que estos deben estar motivados antes, durante y tras el proyecto, ya que serán ellos quienes lo presenten tanto a las familias como al alumnado, y es fundamental que tenga buena acogida de todos los agentes educativos implicados. Para fomentar esto, se han buscado plataformas de código abierto, con interfaces sencillas que tienen tutoriales de manejo para que, además de facilitar la labor docente, el centro escolar y el sistema educativo no tengan que hacer un desembolso económico para poder utilizarlos. Además, al ser de carácter gratuito y sin necesidad de instalación, podrán utilizarse en cualquier dispositivo digital abaratando de este modo el coste de herramientas digitales.

Asimismo, las herramientas finalmente seleccionadas serán de utilidad para el alumnado durante su escolarización y no únicamente durante el proyecto por lo que, además de servirles de ayuda en un momento determinado, le daríamos herramientas TIC útiles a los alumnos y alumnas para continuar con su formación. Además, cuando se acerca al alumnado de modo igualitario a estas plataformas desde edades tempranas, se contribuye a mitigar la brecha digital en cuanto a uso, acceso y formación en TIC que se produce en etapas posteriores (Fernández-Morante et al., 2020).

A través de estas páginas, hemos recogido y desarrollado la idea de trabajar conceptos matemáticos, concretamente geométricos, haciéndose valer de la holografía. Consideramos que en niveles de aplicación básicos el holograma puede ser realizado por el alumnado, permitiéndoles comprender mejor los contenidos dotándolos de significado y volumen. Es por esta razón que, mediante la guía docente, sean las alumnas y alumnos quienes creen y elaboren sus propios hologramas, basados en los conceptos que trabajarán en clase. Y es que, mediante la evaluación del proyecto, hemos podido constatar tanto su viabilidad como los beneficios que reporta.

Creemos oportuno destacar la idea de que con la aplicación de este proyecto el alumno no solo fue capaz de describir el objeto a representar sino también definirlo, ya que como apuntan en González-Regaña et al. (2021) ambos conceptos no son lo mismo. Al tener que elaborar ellos mismos el objeto a representar el docente les da las herramientas para que puedan desarrollarlo, pero sin presentarles físicamente una figura completa. Es decir, el docente les ofrece la descripción de los elementos por los que está compuesta esa figura geométrica y son ellos y ellas quienes han de reorganizar esa información, esa imagen mental, para poder trasladarla al programa informático y crear así la figura geométrica en 3D que corresponda con esa descripción. Ahora bien, tal y como apunta Brea (2008), esta imagen electrónica se recarga de potencial mental, ya que para definir un cuerpo debemos entender qué es y, si esto se conoce, se podrá representar. A fin de cuentas, conocer algo para poder representarlo significa que el sujeto de aprendizaje se apropia intelectualmente del objeto.

Con respecto a la evaluación llevada a cabo durante el proyecto, se constató que es imprescindible una evaluación diagnóstica del alumnado. Esta se entiende como la recogida de "información que permita al docente marcar un punto de partida para emprender un proceso de aprendizaje con sus estudiantes" (Trelles et al., 2017, p. 41). Es por ello que se partirá de lo que el alumnado conozca para no repetir conceptos y no perder así el interés por desarrollar el proyecto 
Del mismo modo, también se da una evaluación formativa. Citando a otros autores, Trelles et al., (2017) apuntan que "no importa tanto valorar los resultados, sino comprender el proceso, supervisarlo e identificar los posibles obstáculos o fallas que pudiera haber en el mismo" (p. 42) con el fin de corregir estas en futuras aplicaciones del proyecto.

A modo de cierre de este trabajo se destacan tres propuestas como objetivos de investigación futura. La primera sería aplicar este proyecto en un aula con alumnado de características parecidas, para saber qué resultados se pueden obtener con la aplicación de este en un número mayor de sujetos. En cuanto a la segunda propuesta, sería interesante observar una progresión del uso de los hologramas no solo en el bloque de la geometría, sino aplicada a otras áreas con la finalidad de acercar elementos a los que no se puede acceder de manera real dentro del aula. Un ejemplo puede ser el estudio de la anatomía interna humana y la estructura de los órganos. Por último, la tercera propuesta sería conocer qué tipo de rol desempeña el profesorado dentro de este modelo de metodología.

\section{REFERENCIAS}

Agra, G., Soares, N., Simplicio, P., Lopes, M.M., Melo, M.D., \& Lima, M.M. (2019). Analysis of the concept of Meaningful Learning in light of the Ausubel's Theory. Revista brasileira de enfermagem, 72(1), 248-255. https://doi.org/10.1590/0034-71672017-0691

Brea, J. L (2008). La era de la imagen electrónica. Contrastes. Revista Internacional De Filosofía, (XIII), 15-28. https://doi. org/10.24310/Contrastescontrastes.v0i0.1268.

Cano, E. (2015). Las rúbricas como instrumento de evaluación de competencias en educación superior: ¿Uso o abuso? Profesorado. Revista de currículum y Formación de profesorado, 19(2), 265-280. https://www.redalyc.org/articulo $.0 a ? \mathrm{id}=56741181017$

Decreto 108/2014, de 4 de julio, del Consell, por el que establece el currículo y desarrolla la ordenación general de la educación primaria en la Comunitat Valenciana. Diari oficial de la Comunitat Valenciana, 7311, de 7 de julio de 2014, pp. 16325-16694. http://www.dogv.gva.es/datos/2014/07/07/ pdf/2014 6347.pdf

Escrivà, M., Jaime, A., \& Gutiérrez, Ál. (2018). Uso de software 3D para el desarrollo de habilidades de visualización en Educación Primaria. Edma 0-6: Educación Matemática en la Infancia $7(1), 42-62$.

Fernández-Morante, C., Cebreiro, B., \& Casal, L. (2020). Capacitar y motivar a las niñas para su participación futura en el sec- tor TIC. Propuesta de cinco países. noeduca. Innoeduca. International Journal of Technology and Educational Innovation, 6(2), 115-127. https://doi.org/10.24310/innoeduca.2020.v6i2.6256

Ghuloum, H. (2010). 3D Hologram Technology in Learning Environment. Informing Sciencie \& IT Education Conference, 10, 693-704

González, A., \& Cerezo, I. (2020). Implicaciones pedagógicas de la realidad aumentada para la mejora de la enseñanza de las ciencias en primaria. Revista Interuniversitaria de Investigación en Tecnología Educativa, (9), 1-16. https:// doi.org/10.6018/riite.444961

González-Regaña, A.J., Martín-Molina, V., Toscano, R., Fernández-León, A., \& Gavilán-Izquierdo, J.M. (2021). El discurso de estudiantes para maestro cuando describen y definen cuerpos geométricos. Enseñanza de las ciencias, 39(1), 8197. https://doi.org/10.5565/rev/ensciencias.3039

Jiménez, A.N., \& Diez-Martínez, E. (2018). Análisis del contenido de apps y videojuegos: implicaciones en procesos cognitivos en la lectura inicial. Apertura, 10(1), 71-87. https:// doi.org/10.32870/ap.v10n1.1114

Kempt, M. (2000). La ciencia del arte. La óptica en el arte occidental de Brunelleschi a Seurat. Akal.

Katsioloudis, P.J., \& Jones, M. V. (2018). A Comparative Analysis of Holographic, 3D-Printed, and Computer-Generated 
Models: Implications for Engineering Technology Students' Spatial Visualization Ability. Journal of Technology Education, 29(2), 36-53. http://doi.org/10.21061/jte.v29i2.a.3

Ley Orgánica 3/2020, de 29 de diciembre, por la que se modifica la Ley Orgánica 2/2006, de 3 de mayo, de Educación (2020). Boletín Oficial del Estado, 340, de 30 de diciembre de 2020, pp. 122868-122953. https://www.boe.es/eli/es/lo/2020/12/29/3

Moncada, J., \& Torres, H. (2016). La coherencia constructivista como estrategia didáctica para el aprendizaje. Revista Educación y Desarrollo Social, 10(2), 50-85. http://dx.doi. $\operatorname{org} / 10.18359 /$ reds. 1775

Navarro, M., Edel, R., \& García, R. I. (2018). Rúbrica para evaluar ambientes virtuales de aprendizaje. 3C TIC. Cuadernos de desarrollo aplicados a las TIC, 7(3), 80-96. http://dx.doi.org/10.1 7993/3ctic.2018.61.80-96/

Orcos, L. (2017). Herramientas holográficas para la enseñanza de la división celular. [Tesis de pregrado]. Universidad de la Rioja, Logroño, España. https://vixra.org/pdf/1704.0065v1.pdf Orcos, L., Jordán Lluch, C., \& Magreñán Ruiz, Á. A. (2018). Uso del holograma como herramienta para trabajar contenidos de geometría en Educación Secundaria. Pensamiento matemático, 8(2), 91-100.

Prensky, M., \& González, V. (2018). Entrevista a Marc Prensky. Revista Interuniversitaria De Investigación En Tecnología Educativa, (5), 12-21. https://doi.org/10.6018/riite/2018/354791

Ribeiro, A., Godoy, G.C., Belini, L., \& Souza-Filho, M. (2018). Holografía y realidad virtual en la enseñanza de nanotecnología: nuevos horizontes dirigidos a educación secundaria. Revista de Física, (56), 34-45.

San Martín, A., \& Peirats, J. (2018). Controversias en la transición del libro de texto en papel y electrónico a los contenidos digitales. Revista de Educación a Distancia, 18(56). https://revistas.um.es/red/article/view/321501

Sánchez, T., Serrano, J.L., \& Rojo, F. (2020). Influencia de la robótica educativa en la motivación y el trabajo cooperativo en Educación Primaria: un estudio de caso. Innoeduca. Interna tional Journal of Technology and Educational Innovation, 6(2), 141-152. https://doi.org/10.24310/innoeduca.2020.v6i2.6779
Sancho, J. (2017). Discursos y prácticas en torno a las competencias en educación. FONSECA: Journal of communcation, (15), 127-144. https://doi.org/10.14201/fjc201715127144

Serra, R., Vega, G., Ferrat, A, Lunazzi. J., \& Magalhàes, D. (2009). El holograma y su utilización como medio de enseñanza de la física en ingeniería. Revista Brasileira de Ensino de Física, 31(1), https://doi.org/10.1590/S1806-11172009000100007.

Trelles, C.A., Bravo, F.E., \& Barrazueta, J.F. (2017). ¿Cómo Evaluar los Aprendizajes en Matemáticas? INNOVA Research Journal, 2(6), 35-51. https://doi.org/10.33890/innova.v2.n6.2017.183

Tonucci, F. (2005). La ciudad de los niños. Un modo nuevo de pensar la ciudad. Fundación Germán Sánchez Ruipérez.

Torres, A. (2020, 29 de mayo). Educación crea por primera vez un comité de matemáticas para rediseñar la asignatura. El País. https://elpais.com/sociedad/2020-05-29/educacion-creapor-primera-vez-un-comite-de-matematicos-para-redisenar-la-asignatura.html

Torres, J. (2015). Organización de los contenidos curriculares y relevancia cultural. En J. Gimeno Sacristán (Ed.), Los contenidos, una reflexión necesaria (pp. 91-102). Ediciones Morata. 